\title{
An Economic Analysis of Sugarcane Cultivation in Ghazipur District of Uttar Pradesh, India
}

\author{
Ashutosh Kumar Ranjan ${ }^{1}$, R. R. Kushwaha ${ }^{1 *}$, Supriya ${ }^{1}$, R. R. Verma ${ }^{1}$, \\ Vinay Kumar Singh ${ }^{2}$, Randhir Yadav ${ }^{1}$ and Ram Singh Yadav ${ }^{1}$ \\ ${ }^{I}$ Department of Agricultural Economics, ${ }^{2}$ Department of Horticulture, Azamgarh Campus, \\ Acharya Narendra Deva University of Agriculture \& Technology, \\ Kumarganj, Ayodhya-224229 (U.P.), India
}

*Corresponding author

\section{A B S T R A C T}

\section{Keywords}

Farm structure, Cropping Pattern, Cropping, Intensity, Cost and Returns etc.

Article Info

Accepted:

11 June 2020 Available Online: 10 July 2020
In the present paper, an attempt has been made to examine various sugarcane production in different categories of the farmers. A study on an economic analysis of sugarcane cultivation in Ghazipur district of eastern Uttar Pradesh was conducted for analysis of the cost of input-output in sugarcane cultivation. A sample of hundred sample farmers (Marginal-55, Small-30, \& Medium-15) was interviewed from few villages of Mardah block of Ghazipur district. Data was analyzed and found that average holding size was 1-18 hectares and cropping intensity was $239.90 \%$. On an average cost of cultivation per hectare was found to be Rs.95124.68. The gross income and net income were found to be Rs.146587.00 and 63156.54 per hectare on overall farms respectively the input output ratio was found to be $1: 1.67$ on Cost C. Sugarcane cultivation in the study was characterized by decreasing returns to scale.

\section{Introduction}

Agriculture is one of the most important sector of Indian economy. It is the only means of living for almost two third population of the India. The agriculture sector of India has occupied $43 \%$ of India's geographical area and contributes about $14 \%$ of Indian GDP. Numbers of crops were grown by the farmers. These include different food crops, commercial crop, oil seed etc. Sugarcane (Saccharum officinarum L.) belongs to family gramineae and originated from tropical south Asia and south east- Asia.

Sugarcane is a renewable, natural agriculture resource because it provides sugar besides biofuel, fibre, fertilizer and myriad of byproducts with ecological sustainability. Sugarcane juice is used for making white 
sugar, brown sugar (Khandhasari), jaggery (Gur) and ethanol. Sugarcane is grown in diversified climatic conditions. India is one of the largest sugarcane producers in the world, producing around 300 million tonnes of cane per annum. Production of sugar is the second largest agro processing industry in the country after cotton and textiles. India is the only country that produces plantation while sugar unlike other countries that produce raw or refined sugar or both. In India, about more than 50 million farmers, are dependent and a large number of agricultural labourers are involved in sugarcane cultivation, harvesting and ancillary activities constituting 7.5 per cent of the rural population and many workers are employed indirectly in processing. Molasses is the chief by-product of sugar industry and is the main raw material for alcohol production and alcohol-based industries in India.

Importance of the crop in the country can be judged from the data during 70's (1970-73) as sugarcane covered an area of 2480 thousand hectares which reached to the 4550 thousand hectare during 2008-11 which was 1.83 times higher than the previous period during 2014 15 sugarcane occupies an area of 5.01 million tonnes with the productivity of $69838 \mathrm{~kg}$ per hectare.

As far as the U.P. state is concerned being commercial crop sugarcane occupied an area of 2.23 million hectares which produce total yield of 135.16 million tonnes having the yield of $60665 \mathrm{~kg}$ per hectare during the year 2014-15 (Directorate of Economics and Statistics Ministry of Agriculture Govt. of India New Delhi 2014-15).

District Ghazipur having best suited agroclimatic condition for sugarcane cultivation have a considerable share in area and production of sugarcane in the state. Sugarcane occupies an area of 7037 hectares and it's total production was 341829 million tonnes the productivity of 485.76 quintals per hectare (District Statistical Bulletin, Ghazipur 2014-15).With this background the study was conducted with the following objectives include to study the farm structure, cropping pattern, and cropping intensity of sample farm. Also to work out the cost and returns of sugarcane production.

\section{Materials and Methods}

\section{Sampling technique}

The purposive and random sampling techniques were used to select, village and farmers. The district Ghazipur was selected purposively. The sampling technique was sub divided into following stages-

Selection of block

Selection of village

Selection of farmers

\section{Selection of block}

At first a list of all blocks of Ghazipur district of Easter Uttar Pradesh along with acreage in sugarcane cultivation were prepared and arranged in descending order, namely "Mardah" block having highest area in sugarcane was selected purposively for this study.

\section{Selection of village}

A list of all villages following "Mardah" block was prepared and arranged in ascending order to take area covered under sugarcane crop and 5(Five) villages were selected randomly from this list.

\section{Selection of farmers}

Three stages satisfied purposive cum random sampling technique was used to select the 
district, block, village and farmers. Ghazipur district of eastern U.P. and Mardah block of district Ghazipur were selected purposively. A list of all the sugarcane growing villages of selected block was prepared and five villages were selected randomly.

A list of all the sugarcane cultivators of each selected village was prepared and arranged in ascending order under three categories i.e.

Marginal (below 1 ha.)

Small (1-2 ha.)

Medium (2-4 ha. and above)

Following proportionate random sampling technique a sample of 100 farmers viz. marginal -55 , small-30 and medium -15 were selected for the purpose of the study.

\section{Method of enquiry}

The primary data information was collected by survey method through personal interview. The data were selected on well structure \& tested schedule but secondary information were option from the tehsil/village and district level official records.

\section{Period of enquiry}

The primary data was collected for the period of one year i.e. Agriculture year 2018-19.

\section{Analytical tools}

Tabular analysis was used for analysis of data weighted average, cropping intensity and cost benefit ratio was worked out with the following formula.

\section{Weighted average}

Where,
WA $=$ Weighted Average, $\mathrm{Xi}=$ Variable

$\mathrm{Wi}=$ Weights of variable

\section{Cropping intensity}

C. I. $=\frac{\text { Total Cropped Area }}{\text { Net Cultivated Area }} \times 100$

Where,

C. I. = cropping intensity

\section{Results and Discussion}

\section{Structure of farms}

Distribution of farm and their cultivated area under different size groups of farms is presented in Table1.1. It is clear from the Table 1.1 that net cultivated area of sample farms constituted $23.38 \%, 32.90 \%$ and $43.72 \%$ sugarcane under marginal small and medium farms respectively.

The average size of land holding of marginal, small and medium farms comes to be 0.5 , 1.29 and 3.42 hectare respectively. On an average holding size was estimated to be 1.18 hectare.

\section{Size of holding}

The average size of holding and cropping is presented in the Table 1.1. It is depicted from the table that average size of holding had increasing trend with increase in the size of farmers. The holding size of marginal, small and medium farms, were found to be $0.5,1.29$ and 3.42 hectare respectively.

\section{Cropping intensity}

Cropping intensity as a ratio between gross cropped area and sown expressed in percentage as presented in the Table 1.2. The cropping intensity on overall farm was found to 239.91 percent which varied from 247.83 
percent at medium size, 229.20 percent at marginal size of farms and it was 240.07 percent at small size of farm respectively. Thereby cropping intensity showed inverse relation with the holding size.

\section{I. $=\frac{\text { Total Cropped Area }}{\text { Net Cultivated Area }} \times 100$}

\section{Cropping pattern}

Cropping pattern is the proportion of area under different crops at a point of time. It is an important factor to decide the level of investment for different input on farm and income of farmer based on resource availability and climatic condition.

\section{Investment of farm assets}

Per form investment by different categories of farms on farm assets like farm building, implement and machinery, irrigation, livestock and other implement structure are presented in Table 1.3. It is revealed from the table that the average expenditure of farm assets on overall farm was Rs. 509200.90.
Major part of this expenditure was incurred on building i.e. 63.24 percent, machinery and implements 24.56 percent, and livestock 12.20 percent respectively.

Farm size group wise investment came to be Rs. 256176.30, Rs. 608449.80and Rs. 871793.11 corresponding to marginal, small and medium size group of farms. It may be concluded that per farm investment on farm assets was having direct relationship with size of holding.

\section{Structure of cost and returns}

Per hectare cost and return from the cultivation of sugarcane crop on different categories of farm were worked out and presented in Table 1.5.

The per hectare cost " $\mathrm{C}_{3}$ " was worked out to be Rs. 91956.74 on marginal, Rs. 94820.23 on small and Rs. 95124 on medium farm with and overall average of Rs. 93290.98 respectively. Cost of production per quintal had the negative relation with the size of farms.

Table.1 Average size of land holding under different size group of sample farms

\begin{tabular}{|c|l|c|c|c|}
\hline $\begin{array}{c}\text { S. } \\
\text { No. }\end{array}$ & \multicolumn{1}{|c|}{\begin{tabular}{c}
\multicolumn{1}{|c|}{$\begin{array}{c}\text { Size of Group } \\
\text { Farms }\end{array}$} \\
\hline $\mathbf{1}$
\end{tabular}} & $\begin{array}{c}\text { No. of } \\
\text { sample } \\
\text { farms }\end{array}$ & $\begin{array}{c}\text { Net } \\
\text { cultivated } \\
\text { area } \\
\text { (below 1 ha) }\end{array}$ & $\begin{array}{c}\text { Averages } \\
\text { size of land } \\
\text { holding }\end{array}$ \\
\hline $\mathbf{2}$ & $\begin{array}{l}\text { Small Farms } \\
\text { (1-2 ha) }\end{array}$ & 55 & $27.50(23.38)$ & $\mathbf{0 . 5}$ \\
\hline $\mathbf{3}$ & $\begin{array}{l}\text { Medium Farms } \\
\text { (2-4 ha \& above) }\end{array}$ & 30 & $38.70(32.90)$ & $\mathbf{1 . 2 9}$ \\
\hline & \multicolumn{1}{|c|}{ Total } & 15 & $51.42(43.72)$ & $\mathbf{3 . 4 2}$ \\
\hline
\end{tabular}


Table.2 Per farm investment on different size group of sample farms (Rs.)

\begin{tabular}{|c|c|c|c|c|c|}
\hline \multirow{2}{*}{$\begin{array}{l}\text { S. } \\
\text { No. }\end{array}$} & \multirow[t]{2}{*}{ Particulars } & \multicolumn{4}{|c|}{ Size of farms } \\
\hline & & Marginal (55) & Small (30) & $\begin{array}{l}\text { Medium } \\
\text { (15) }\end{array}$ & $\begin{array}{c}\text { Overall } \\
\text { average }\end{array}$ \\
\hline 1. & Buildings & $\begin{array}{c}232819.2 \\
(65.37)\end{array}$ & $\begin{array}{c}348181.55 \\
(57.22)\end{array}$ & $\begin{array}{c}596728.77 \\
(68.45)\end{array}$ & $\begin{array}{c}322014.3 \\
(63.24)\end{array}$ \\
\hline \multirow[t]{3}{*}{ I. } & Residential & $\begin{array}{c}217915.39 \\
(61.18)\end{array}$ & $\begin{array}{c}326381.69 \\
(53.64)\end{array}$ & $\begin{array}{c}571861.53 \\
(65.6)\end{array}$ & $\begin{array}{r}303547.2 \\
(59.61)\end{array}$ \\
\hline & a. Kachcha & $\begin{array}{c}10416.54 \\
(2.93)\end{array}$ & $\begin{array}{c}7771.42 \\
(1.28)\end{array}$ & $\begin{array}{c}7984.61 \\
(0.91)\end{array}$ & $\begin{array}{c}9282.965 \\
(1.823)\end{array}$ \\
\hline & b.Pacca & $\begin{array}{c}207453.85 \\
(58.24)\end{array}$ & $\begin{array}{c}318610.27 \\
(52.36)\end{array}$ & $\begin{array}{c}563876.92 \\
(64.68)\end{array}$ & $\begin{array}{r}294264.2 \\
(57.79)\end{array}$ \\
\hline \multirow[t]{3}{*}{ II. } & Cattle shed & $\begin{array}{c}14903.76 \\
(4.18)\end{array}$ & $\begin{array}{c}21799.86 \\
(3.58)\end{array}$ & $\begin{array}{c}24867.24 \\
(2.85)\end{array}$ & $\begin{array}{c}18467.11 \\
(3.627)\end{array}$ \\
\hline & a. Kachcha & $\begin{array}{c}12355.76 \\
(3.46)\end{array}$ & $\begin{array}{c}6514.28 \\
(1.07)\end{array}$ & $\begin{array}{c}4938.56 \\
(0.56)\end{array}$ & $\begin{array}{c}9490.736 \\
(1.864)\end{array}$ \\
\hline & b.Pacca & $\begin{array}{c}2548.00 \\
(0.71)\end{array}$ & $\begin{array}{c}15285.58 \\
(2.51)\end{array}$ & $\begin{array}{c}19928.68 \\
(2.28)\end{array}$ & $\begin{array}{r}8976.376 \\
(1.763)\end{array}$ \\
\hline \multirow[t]{4}{*}{2.} & Live stock & $\begin{array}{c}59590.75 \\
(16.73)\end{array}$ & $\begin{array}{c}67677.48 \\
(11.12)\end{array}$ & $\begin{array}{c}60430.6 \\
(6.93)\end{array}$ & $\begin{array}{c}\text { 62142.74 } \\
\text { (12.2) }\end{array}$ \\
\hline & a. Cow & $\begin{array}{c}19173.07 \\
(5.38)\end{array}$ & $\begin{array}{c}13429.96 \\
(2.20)\end{array}$ & $\begin{array}{c}12080.00 \\
(1.38)\end{array}$ & $\begin{array}{c}16386.18 \\
(3.218)\end{array}$ \\
\hline & b.Buffalo & $\begin{array}{c}39490.76 \\
(11.09)\end{array}$ & $\begin{array}{c}53008.00 \\
(8.71)\end{array}$ & $\begin{array}{c}47918.58 \\
(5.49)\end{array}$ & $\begin{array}{c}44810.11 \\
(8.8)\end{array}$ \\
\hline & c. Goat & $\begin{array}{l}926.92 \\
(0.26)\end{array}$ & $\begin{array}{c}1239.52 \\
(0.20)\end{array}$ & $\begin{array}{c}432.00 \\
(0.05)\end{array}$ & $\begin{array}{c}946.462 \\
(0.186)\end{array}$ \\
\hline 3. & $\begin{array}{l}\text { Machinery and } \\
\text { Implement }\end{array}$ & $\begin{array}{c}63766.39 \\
(17.9)\end{array}$ & $\begin{array}{c}192590.78 \\
(31.65)\end{array}$ & $\begin{array}{c}214633.76 \\
(24.62)\end{array}$ & $\begin{array}{l}125043 \\
(24.56)\end{array}$ \\
\hline I. & Minor Implement & $\begin{array}{l}865.87 \\
(0.24)\end{array}$ & $\begin{array}{l}615.99 \\
(0.10)\end{array}$ & $\begin{array}{l}791.38 \\
(0.09)\end{array}$ & $\begin{array}{c}779.7325 \\
(\mathbf{0 . 1 5 3})\end{array}$ \\
\hline II. & Major Implement & $\begin{array}{c}62900.52 \\
(17.66)\end{array}$ & $\begin{array}{c}191974.79 \\
(31.55)\end{array}$ & $\begin{array}{c}213842.38 \\
(24.53)\end{array}$ & $\begin{array}{c}124264.1 \\
(24.4)\end{array}$ \\
\hline 4. & Grand total & $\begin{array}{c}356176.3 \\
(100)\end{array}$ & $\begin{array}{c}608449.8 \\
(100)\end{array}$ & $\begin{array}{c}871793.11 \\
(100)\end{array}$ & $\begin{array}{c}509200.9 \\
(100)\end{array}$ \\
\hline
\end{tabular}


Table.3 Cropping Pattern under different size group of sample farms (Area in ha and percentage)

\begin{tabular}{|c|c|c|c|c|c|}
\hline \multirow[t]{2}{*}{ S.No. } & \multirow[t]{2}{*}{ Name of Crops } & \multicolumn{3}{|c|}{ Average size of sample farms } & \multirow{2}{*}{$\begin{array}{c}\text { Overall } \\
\text { average }\end{array}$} \\
\hline & & Marginal & Small & Medium & \\
\hline A. & Kharif & $\begin{array}{c}0.500 \\
(43.63)\end{array}$ & $\begin{array}{c}1.288 \\
(41.59)\end{array}$ & $\begin{array}{c}3.411 \\
(40.24)\end{array}$ & $\begin{array}{c}1.173 \\
(41.40)\end{array}$ \\
\hline 1 & Paddy & $\begin{array}{c}0.256 \\
(22.34)\end{array}$ & $\begin{array}{c}0.538 \\
(17.37)\end{array}$ & $\begin{array}{c}1.357 \\
(16.01)\end{array}$ & $\begin{array}{c}\mathbf{0 . 5 0 6} \\
(17.90)\end{array}$ \\
\hline 2 & Maize & $\begin{array}{c}0.125 \\
(10.91)\end{array}$ & $\begin{array}{c}0.399 \\
(12.88)\end{array}$ & $\begin{array}{c}1.025 \\
(12.09)\end{array}$ & $\begin{array}{c}\mathbf{0 . 3 4 2} \\
(\mathbf{1 2 . 1 0})\end{array}$ \\
\hline 3 & Pigeon Pea & $\begin{array}{l}0.039 \\
(3.40)\end{array}$ & $\begin{array}{l}0.063 \\
(2.03)\end{array}$ & $\begin{array}{l}0.108 \\
(1.27)\end{array}$ & $\begin{array}{l}\mathbf{0 . 0 5 7} \\
(\mathbf{2 . 0 0})\end{array}$ \\
\hline 4 & Urd & $\begin{array}{l}0.030 \\
(2.62)\end{array}$ & $\begin{array}{l}0.080 \\
(2.58)\end{array}$ & $\begin{array}{l}0.599 \\
(7.07)\end{array}$ & $\begin{array}{l}\mathbf{0 . 1 3 0} \\
(\mathbf{4 . 6 0})\end{array}$ \\
\hline 5 & Chari & $\begin{array}{l}0.025 \\
(2.18)\end{array}$ & $\begin{array}{l}0.108 \\
(3.49)\end{array}$ & $\begin{array}{l}0.150 \\
(1.77)\end{array}$ & $\begin{array}{l}\mathbf{0 . 0 6 9} \\
(2.42)\end{array}$ \\
\hline 6 & Vegetable & $\begin{array}{l}0.025 \\
(2.18)\end{array}$ & $\begin{array}{l}0.100 \\
(3.23)\end{array}$ & $\begin{array}{l}0.172 \\
(2.03)\end{array}$ & $\begin{array}{l}\text { 0.070 } \\
(2.46)\end{array}$ \\
\hline B. & Rabi & $\begin{array}{c}0.461 \\
(40.23)\end{array}$ & $\begin{array}{c}1.227 \\
(39.62)\end{array}$ & $\begin{array}{c}3.32 \\
(39.17)\end{array}$ & $\begin{array}{c}1.120 \\
(39.50)\end{array}$ \\
\hline 1 & Wheat & $\begin{array}{c}0.256 \\
(22.34)\end{array}$ & $\begin{array}{c}0.538 \\
(17.37)\end{array}$ & $\begin{array}{c}1.357 \\
(16.01)\end{array}$ & $\begin{array}{c}\mathbf{0 . 5 0 6} \\
(\mathbf{1 7 . 9 0})\end{array}$ \\
\hline 2 & Mustard & $\begin{array}{l}0.056 \\
(4.89)\end{array}$ & $\begin{array}{c}0.316 \\
(10.20)\end{array}$ & $\begin{array}{c}1.025 \\
(12.09)\end{array}$ & $\begin{array}{l}0.279 \\
(9.87)\end{array}$ \\
\hline 3 & Lentil & $\begin{array}{l}0.020 \\
(1.75)\end{array}$ & $\begin{array}{l}0.107 \\
(3.45)\end{array}$ & $\begin{array}{l}0.125 \\
(1.47)\end{array}$ & $\begin{array}{l}0.062 \\
(2.18)\end{array}$ \\
\hline 4 & Pea & $\begin{array}{l}0.075 \\
(6.55)\end{array}$ & $\begin{array}{l}0.068 \\
(2.19)\end{array}$ & $\begin{array}{c}0.445 \\
(5.025)\end{array}$ & $\begin{array}{l}0.128 \\
(4.54)\end{array}$ \\
\hline 5 & Berseem & $\begin{array}{l}0.029 \\
(2.53)\end{array}$ & $\begin{array}{l}0.098 \\
(3.16)\end{array}$ & $\begin{array}{l}0.196 \\
(2.31)\end{array}$ & $\begin{array}{l}\mathbf{0 . 0 7 5} \\
(\mathbf{2 . 6 4})\end{array}$ \\
\hline 6 & Vegetable & $\begin{array}{l}0.025 \\
(2.18)\end{array}$ & $\begin{array}{l}0.100 \\
(3.23)\end{array}$ & $\begin{array}{l}0.172 \\
(2.03)\end{array}$ & $\begin{array}{l}\mathbf{0 . 0 7 0} \\
(2.46)\end{array}$ \\
\hline C. & Zaid & $\begin{array}{c}0.185 \\
(16.14)\end{array}$ & $\begin{array}{c}0.582 \\
(18.79)\end{array}$ & $\begin{array}{c}1.745 \\
(20.59)\end{array}$ & $\begin{array}{c}\mathbf{0 . 5 3 8} \\
(\mathbf{1 9 . 0 0 )}\end{array}$ \\
\hline 1 & Sugarcane & $\begin{array}{c}0.131 \\
(11.43)\end{array}$ & $\begin{array}{c}0.348 \\
(12.40)\end{array}$ & $\begin{array}{c}1.470 \\
(17.34)\end{array}$ & $\begin{array}{c}\mathbf{0 . 4 0 8} \\
(\mathbf{1 4 . 4 0 )}\end{array}$ \\
\hline 2 & Chari & $\begin{array}{l}0.029 \\
(2.53)\end{array}$ & $\begin{array}{l}0.098 \\
(3.16)\end{array}$ & $\begin{array}{l}0.150 \\
(1.77)\end{array}$ & $\begin{array}{l}\mathbf{0 . 0 6 8} \\
(2.40)\end{array}$ \\
\hline 3 & Vegetable & $\begin{array}{l}0.025 \\
(2.18)\end{array}$ & $\begin{array}{l}0.100 \\
(3.23)\end{array}$ & $\begin{array}{l}0.125 \\
(1.47)\end{array}$ & $\begin{array}{l}0.063 \\
(2.21)\end{array}$ \\
\hline \multicolumn{2}{|c|}{ Total $(A+B+C)$} & $\begin{array}{l}1.146 \\
(100)\end{array}$ & $\begin{array}{l}3.097 \\
(100)\end{array}$ & $\begin{array}{l}8.476 \\
(100)\end{array}$ & $\begin{array}{l}2.831 \\
(100)\end{array}$ \\
\hline
\end{tabular}

Figure in parentheses indicate the percentage to the total cropped area 
Table.4 Cropping intensity of different size group of sample farms (\%)

\begin{tabular}{|c|l|c|c|c|c|}
\hline $\begin{array}{c}\text { S. } \\
\text { No. }\end{array}$ & $\begin{array}{c}\text { Size group of } \\
\text { farms }\end{array}$ & No. of farms & $\begin{array}{c}\text { Net cultivated } \\
\text { area (ha) }\end{array}$ & $\begin{array}{c}\text { Gross Cropped } \\
\text { area (ha) }\end{array}$ & $\begin{array}{c}\text { Cropping } \\
\text { intensity }\end{array}$ \\
\hline 1. & Marginal & 55 & 0.50 & 1.146 & $\mathbf{2 2 9 . 2}$ \\
\hline 2. & Small & 30 & 1.29 & 3.097 & $\mathbf{2 4 0 . 0 7}$ \\
\hline 3. & Medium & 15 & 3.42 & 8.476 & $\mathbf{2 4 7 . 8 3}$ \\
\hline & Average & $\mathbf{1 0 0}$ & $\mathbf{1 . 1 8}$ & $\mathbf{2 . 8 3 1}$ & $\mathbf{2 3 9 . 9 1}$ \\
\hline
\end{tabular}

Table.5 Per hectare costs and income of sugarcane cultivation (Rs.)

\begin{tabular}{|c|c|c|c|c|c|c|c|c|c|}
\hline \multirow{3}{*}{$\begin{array}{l}\text { S. No. } \\
\text { A. }\end{array}$} & \multirow{3}{*}{$\begin{array}{l}\text { Particulars } \\
\text { Cost } \\
\text { expenditure }\end{array}$} & \multicolumn{8}{|c|}{ Size group of farms } \\
\hline & & \multicolumn{2}{|c|}{ Marginal } & \multicolumn{2}{|c|}{ Small } & \multicolumn{2}{|c|}{ Medium } & \multicolumn{2}{|c|}{ Overall average } \\
\hline & & Rs. & $\%$ & Rs. & $\%$ & Rs. & $\%$ & Rs. & $\%$ \\
\hline 1. & $\begin{array}{l}\text { Human } \\
\text { Labour }\end{array}$ & 15120.87 & 16.44 & 15674.34 & 16.53 & 12721.51 & 16.53 & 15377.01 & 16.48 \\
\hline a. & $\begin{array}{l}\text { Family } \\
\text { Labour }\end{array}$ & 9831.39 & 10.69 & 5791.72 & 6.11 & 4891.12 & 5.14 & 7878.44 & 8.44 \\
\hline b. & $\begin{array}{l}\text { Hired } \\
\text { Labour }\end{array}$ & 5289.48 & 5.75 & 9882.62 & 10.42 & 1083039 & 11.39 & 7498.55 & 8.03 \\
\hline 2. & $\begin{array}{l}\text { Machinery } \\
\text { Power }\end{array}$ & 5796.58 & 6.30 & 5808.16 & 6.12 & 6291.87 & 6.61 & 5874.34 & 6.297 \\
\hline 3. & $\begin{array}{l}\text { Seed and } \\
\text { showing }\end{array}$ & 13069.98 & 14.21 & 13869.84 & 14.63 & 13900.79 & 14.61 & 13434.56 & 14.4 \\
\hline 4. & $\begin{array}{l}\text { Manure and } \\
\text { fertilizer }\end{array}$ & 13008.31 & 14.15 & 13880.54 & 14.64 & 13329.58 & 14.01 & 13318.17 & 14.28 \\
\hline 5. & Irrigation & 10898.69 & 11.85 & 10968.22 & 11.57 & 11009.68 & 11.57 & 10936.20 & 11.72 \\
\hline 6. & $\begin{array}{l}\text { Plant } \\
\text { protection }\end{array}$ & 856.31 & 0.93 & 885.86 & 0.93 & 890.62 & 0.93 & 870.32 & 0.93 \\
\hline 7. & $\begin{array}{l}\text { Total } \\
\text { working } \\
\text { capital }\end{array}$ & 48919.35 & 5302 & 55295.24 & 58.32 & 56252.93 & 59.14 & 51932.15 & 55.67 \\
\hline 8. & $\begin{array}{l}\text { Interest on } \\
\text { working } \\
\text { capital }\end{array}$ & 1956.77 & 2.12 & 2211.81 & 2.33 & 2250.11 & 2.36 & 2077.28 & 2.22 \\
\hline 9. & $\begin{array}{l}\text { Rental value } \\
\text { of land }\end{array}$ & 18000.00 & 19.57 & 18000.00 & 18.98 & 18000.00 & 18.92 & 18000.00 & 19.29 \\
\hline 10. & $\begin{array}{l}\text { Interest on } \\
\text { fixed capital }\end{array}$ & 4889.53 & 5.31 & 4901.44 & 5.17 & 5082.63 & 5.34 & 4922.06 & 5.27 \\
\hline 11. & Sub Total & 83597.04 & 90.91 & 86200.21 & 90.91 & 86476.99 & 90.91 & 84809.98 & 90.91 \\
\hline 12. & $\begin{array}{l}\text { Marginal } \\
\text { Cost@ } 10 \% \\
\text { of sub-total }\end{array}$ & 8359.70 & 9.09 & 8620.02 & 9.09 & 8647.69 & 9.09 & 8480.99 & 9.09 \\
\hline \multicolumn{2}{|c|}{ Grand Total } & 91956.74 & 100 & 94820.23 & 100 & 95124.68 & 100 & 93290.98 & 100 \\
\hline
\end{tabular}


Table.6 Measures of per hectare cost and return of sugarcane (Rs.)

\begin{tabular}{|c|c|c|c|c|c|c|c|}
\hline \multirow{3}{*}{$\begin{array}{c}\text { S.No. } \\
\text { B. }\end{array}$} & \multirow{2}{*}{\multicolumn{3}{|c|}{ Particulars }} & \multicolumn{3}{|c|}{ Size Group of Farms } & \multirow[t]{3}{*}{ Overall Average } \\
\hline & & & & \multirow{2}{*}{ Marginal } & \multirow[t]{2}{*}{ Small } & \multirow{2}{*}{ Medium } & \\
\hline & \multicolumn{3}{|c|}{ Income } & & & & \\
\hline 1 & \multicolumn{3}{|c|}{ Cost A1/A2 } & 50876.12 & 57507.05 & 58503.04 & 54009.44 \\
\hline 2 & \multicolumn{3}{|c|}{ Cost B1 } & 55765.65 & 62408.49 & 63585.87 & 58931.54 \\
\hline 3 & \multicolumn{3}{|c|}{ Cost B2 } & 73765.65 & 80408.49 & 81585.87 & 76931.54 \\
\hline 4 & \multicolumn{3}{|c|}{ Cost $\mathrm{C} 1$} & 65597.04 & 68200.21 & 68476.99 & 66809.98 \\
\hline 5 & \multicolumn{3}{|c|}{ Cost $\mathrm{C}_{2}$} & 83597.04 & 86200.21 & 86476.99 & 84809.98 \\
\hline 6 & \multicolumn{3}{|c|}{ Cost $\mathrm{C} 3$} & 91956.74 & 94820.23 & 95124.68 & 93290.98 \\
\hline \multirow[t]{2}{*}{7} & \multirow[t]{2}{*}{ Yield (q/ha.) } & a. & Main Product & 520.90 & 525.80 & 528.60 & 523.53 \\
\hline & & b. & By-product & 120.00 & 126.38 & 128.95 & 123.26 \\
\hline \multirow{3}{*}{8} & \multirow{3}{*}{ Gross Income (₹ ) } & a. & Main Product & 145852.00 & 147224.00 & 148008.00 & 146587.00 \\
\hline & & b. & By Product & 9600.00 & 10110.40 & 10316.00 & 9860.52 \\
\hline & & c. & Total & 155452.00 & 157334.40 & 158324.00 & 156447.50 \\
\hline 9 & \multicolumn{3}{|c|}{ Net Return over Cost C3 } & 63495.26 & 62514.17 & 63199.32 & 63156.54 \\
\hline 10 & \multicolumn{3}{|c|}{ Family income } & 81686.35 & 76925.91 & 76738.13 & 59515.99 \\
\hline 11 & \multicolumn{3}{|c|}{ Farm Business Income } & 104575.88 & 99827.35 & 99820.96 & 102438.10 \\
\hline 12 & \multicolumn{3}{|c|}{ Farm Investment Income } & 22889.53 & 22901.44 & 23082.83 & 22922.10 \\
\hline 13 & \multicolumn{3}{|c|}{ Cost of Production (q/ha.) } & 164.22 & 167.98 & 167.96 & 165.91 \\
\hline \multirow{6}{*}{14} & \multirow{6}{*}{$\begin{array}{l}\text { Benefit: Cost } \\
\text { (B:C) Ratio }\end{array}$} & a. & On the Basis of Cost A1 & $1: 3.05$ & $1: 2.73$ & $1: 2.70$ & $1: 2.89$ \\
\hline & & b. & On the Basis of Cost B1 & $1: 2.78$ & $1: 2.52$ & $1: 2.48$ & $1: 2.65$ \\
\hline & & c. & On the Basis of Cost B2 & $1: 2.10$ & $1: 1.95$ & $1: 1.94$ & $1: 2.03$ \\
\hline & & d. & On the Basis of Cost $\mathrm{C} 1$ & $1: 2.36$ & $1: 2.30$ & $1: 2.31$ & $1: 2.34$ \\
\hline & & e. & On the Basis of Cost $\mathrm{C}_{2}$ & $1: 1.85$ & $1: 1.82$ & $1: 1.83$ & $1: 1.84$ \\
\hline & & f. & On the Basis of Cost $\mathrm{C}_{3}$ & 1:1.69 & $1: 1.65$ & 1:1.66 & $1: 1.17$ \\
\hline
\end{tabular}


The overall size of holding in the study area was $0.50,1.29$ and 3.42 hectare in marginal, small and medium size of farms respectively. It was found that medium farmers were cultivating maximum area followed by small and marginal categories of farms. The cropping intensity was 239.91 percent on an overall average. The cropping intensity decreases with increase in the size of holding.

Per farm average investment came to be Rs. 356176.30, Rs. 608449.80 and Rs. 871793.11 corresponding to marginal, small and medium size group of farms. It may be able to be conducted that per farm investment on farm assets was having direct relationship with size of holding. Gross income, net income, farm business income, family labour income and farm investment income shows the positive relationship with the size of farms. The per quintal cost of production of sugarcane on overall farm was Rs. 165.91 whereas cost of production in (Rs./qtl.) came out to be Rs. 164.22, Rs. 167.98 and 167.96 for marginal, small and medium farms respectively.

\section{References}

Keshavainh, K.V., Palled, Y.B. and Nandihalli, B.S. (2013) Economics of sugarcane cultivation under organic and integrated nutrient management practices in Cauvery Command Area. Research Journal of crop Improvement. 4(2):127-130.

Murthy, P.S.R. (1999) Economics of sugarcane cultivation in West Godavari district of Andhra Pradesh - a review. CAB Abstracts Economics Affairs (Calcutta),44(3):172-176.

Peykani, G. R. Kelashemi, M. K. Shahbazi, H. and Akrami, A. H. (2010). Determination of suitable sugarcane utilization system using Total Factor Productivity (TFP) (case study: Imam Khomeini Cultivation of Processing Center in Khuzestan Province). Journal of agricultural Science and Technology. 12:511-521.

Singh, J. P. (2006). Prospects for reducing cost of cultivation of sugarcane and measures to improve sugarcane yield and sugar recovery. Co-operative Sugar.38 (2):33-43

Verma, R. P. and Verma, R. S. (1996). Assessment of economics and adoption feasibility of improved sugarcane beads cropping system under real farming situation of north India. Indian Journal of Technology.11(1):46-56.

\section{How to cite this article:}

Ashutosh Kumar Ranjan, R. R. Kushwaha, Supriya, R. R. Verma, Vinay Kumar Singh, Randhir Yadav and Ram Singh Yadav. 2020. An Economic Analysis of Sugarcane Cultivation in Ghazipur District of Uttar Pradesh, India. Int.J.Curr.Microbiol.App.Sci. 9(07): 945-953. doi: https://doi.org/10.20546/ijcmas.2020.907.110 